\title{
Imaging Collective Magnonic Modes in 2D Arrays of Magnetic Nanoelements
}

\author{
V. V. Kruglyak, ${ }^{1, *}$ P. S. Keatley, ${ }^{1}$ A. Neudert, ${ }^{1}$ R. J. Hicken, ${ }^{1}$ J. R. Childress,${ }^{2}$ and J. A. Katine ${ }^{2}$ \\ ${ }^{1}$ School of Physics, University of Exeter, Stocker Road, Exeter, EX4 4QL, United Kingdom \\ ${ }^{2}$ Hitachi Global Storage Technologies, San Jose Research Center, San Jose, California 95135, USA
}

(Received 19 August 2009; published 14 January 2010)

\begin{abstract}
We have used time resolved scanning Kerr microscopy to image collective spin wave modes within a 2D array of magnetic nanoelements. Long wavelength spin waves are confined within the array as if it was a continuous element of the same size but with effective material properties determined by the structure of the array and its constituent nanoelements. The array is an example of a magnonic metamaterial, the demonstration of which provides new opportunities within the emerging field of magnonics.
\end{abstract}

DOI: 10.1103/PhysRevLett.104.027201

When oscillators are made to interact, i.e., they are "coupled," their dynamics are no longer described by the frequencies of the individual oscillators [1]. Instead, the motion of each oscillator is characterized by the same set of new frequencies of the so called "normal" (or "collective") modes. Each of the collective modes can be individually excited, but none of the coupled oscillators can move without exciting motion of the others. For each normal mode, a constant amplitude and phase relationship is maintained among the coupled oscillators irrespective of the excitation. For example, for a system of two coupled oscillators, so called "acoustic" and "optical" modes are characterized by in-phase and out-of-phase motion of the oscillators, respectively. Examples of excitations in systems containing a larger number of coupled oscillators are vibrational and spin waves, representing collective modes of large arrays of atoms [2] and spins [3], respectively.

Magnetization dynamics in arrays of magnetic elements have been studied mainly with the aim of exploring the properties of the magnetic material and the spin wave modes confined within individual elements $[4,5]$. The interaction among elements within arrays has been considered as an inevitable yet small obstacle for such studies. Recently the emphasis of such research has shifted to the collective dynamic magnetic phenomena themselves [6,7]. This is due in part to the realization that the coupling might become a limiting factor in the application of bit patterned magnetic recording at high recording densities [8]. From another perspective, interest has been fuelled by opportunities for exploitation of the coupling, e.g., for phase locking of arrays of spin transfer torque nano-oscillators [9] or for creation of magnonic crystals [10], the latter being the central object of the emerging field of magnonics-the study of spin waves in magnetic nanostructures [11].

Time resolved scanning Kerr microscopy (TRSKM) has been used to image nonuniform dynamics in magnetic microelements [12-15] and to measure the average response from magnetic nanoelements within micron sized arrays [5]. However, no imaging of collective spin wave modes in closely packed arrays of magnetic nanoelements has been demonstrated. In this Letter, we present the first
PACS numbers: 75.30.Ds, 75.40.Gb, 75.75.-c, 78.20.Ls

TRSKM images of collective magnonic modes in a two dimensional (2D) array of nanoelements. The array behaves as a quasicontinuous "magnonic" metamaterial, with properties defined by the geometry of the array, that supports long wavelength collective spin waves.

Specifically, we studied a $\sim 4 \times 4 \mu \mathrm{m}^{2}$ array of $\sim 80 \times$ $40 \mathrm{~nm}^{2}$ "stadium" shaped elements with $\sim 20 \mathrm{~nm}$ edge-toedge separation. The sample was fabricated from a $\mathrm{Co}_{50} \mathrm{Fe}_{50}(0.7 \mathrm{~nm}) / \mathrm{Ni}_{92} \mathrm{Fe}_{8}(4.5 \mathrm{~nm})$ film [16,17] on top of the $30 \mu \mathrm{m}$ wide central conductor of a $50 \Omega$ coplanar waveguide (CPW). The latter was used to deliver $\sim 7 \mathrm{~V}$ pulses of $\sim 70$ ps duration from an electronic pulse generator to the sample. The voltage pulses in turn created $\sim 6$ Oe pulses of in-plane magnetic field at the sample position. The train of magnetic pulses was synchronized to a Ti:Sapphire laser producing $100 \mathrm{fs}$ optical probe pulses of $800 \mathrm{~nm}$ wavelength at the same repetition rate of $80 \mathrm{MHz}$. A microscope objective was used to focus the linearly polarized optical pulses to a $\sim 800 \mathrm{~nm}$ spot. The train of magnetic pulses was amplitude (on/off) modulated at $\mathrm{kHz}$ frequency, and the polarization rotation of the reflected optical pulse due to the polar Kerr effect was measured using an optical bridge detector and a lock-in amplifier. The measured signal was proportional to the outof-plane component of the dynamic magnetization averaged over an ensemble of elements within the probed region. To acquire time resolved (TR) signals, the time delay between the optical and magnetic pulses was varied for a fixed position of the optical spot on the surface of the sample. To acquire images of the dynamic magnetization at a fixed time delay, a piezoelectric scanning stage was used to move the objective lens and thereby to scan the position of the optical spot. An in-plane static magnetic field was applied parallel to the CPW and to the long axis of the nanoelements, as shown in Fig. 1(c).

Measurements were first performed with the laser spot focused in the center of the array. The acquired oscillatory TR signals [Fig. 1(a)] exhibit a slow modulation of the oscillation amplitude at the absolute values of the bias field smaller than 400 Oe. The oscillatory signal persists when the sign of the bias field is changed, and its phase flips by 




FIG. 1. (a) TR signals acquired from the array are shown for different values and the history of the bias magnetic field. The "+" and "-" signs in the parenthesis correspond to the bias field approached from saturation at large positive and negative magnetic fields, respectively. (b) FFT spectra of the TR signals from (a) are shown for several values of the bias field approached from positive saturation. (c) A scanning electron microscope image of a portion of the array is shown together with the directions of the bias $\left(\mathbf{H}_{\text {bias }}\right)$ and pulsed $\left(\mathbf{H}_{\text {pulsed }}\right)$ magnetic fields.

$180^{\circ}$ between -28 and -79 Oe, which corresponds to the reversal of the direction of the static magnetization. No signal was detected at -53 Oe, which was most likely due to a distribution of switching fields for different elements within the array that led to cancellation of the response from switched and not switched elements. The same behavior was observed when the bias field was varied after starting from saturation at a large negative field. Figure 1(b) presents the fast Fourier transform (FFT) spectra calculated from some of the TR signals shown in Fig. 1(a). The main feature of the spectra is the splitting of the broad single peak observed at higher bias field values into the three narrower peaks that are just resolved at field values at which the beating was observed in the TR signals shown in Fig. 1(a).

To explore the origin of this splitting, we used the object-oriented micromagnetic framework (OOMMF) [18] to simulate the magnetization dynamics within both an isolated nanoelement and an array of $3 \times 3$ nanoelements $[5,19]$, which had size and shape of those within the measured array. The sample was divided into square $1 \mathrm{~nm}$ cells with height equal to the thickness of the sample. The hysteresis loop was simulated first. Then, the static magnetization state at a particular value of the static magnetic field was used as the initial configuration for dynamic simulations, in which the pulsed magnetic field was applied to the sample, as in the experiment. The dynamic magnetic state was recorded every 20 ps during the first
$10 \mathrm{~ns}$ after excitation. To determine the spatial character of the excited modes, a TR trace was generated from the outof-plane component of the magnetization at each pixel and Fourier transformed. The FFT spectra were used to reconstruct images of the magnitude and phase of the dynamic magnetization ("mode images") at frequencies of particular resonant modes that had been identified from the FFT spectra calculated from the average TR response of either the center element of the $3 \times 3$ array or the isolated element $[14,20,21]$.

Figure 2(a) shows FFT spectra calculated from the average dynamic magnetization of the isolated nanoelement. The spectra contain two peaks, attributable to the "edge" and "delocalized" modes [5,22]. In the latter mode observed at a higher frequency, the magnetization in the center and the ends of the element precesses out-of-phase, resulting in a greatly reduced observable signal. Hence, here, we will concentrate on the low frequency (edge) mode.

Figure 2(b) shows the low frequency part of the FFT spectra calculated from the average dynamic magnetization of the center element within the $3 \times 3$ array. Several peaks are generally observed in the spectra. As shown in Fig. 3 for the bias field of 197 Oe, the mode images for

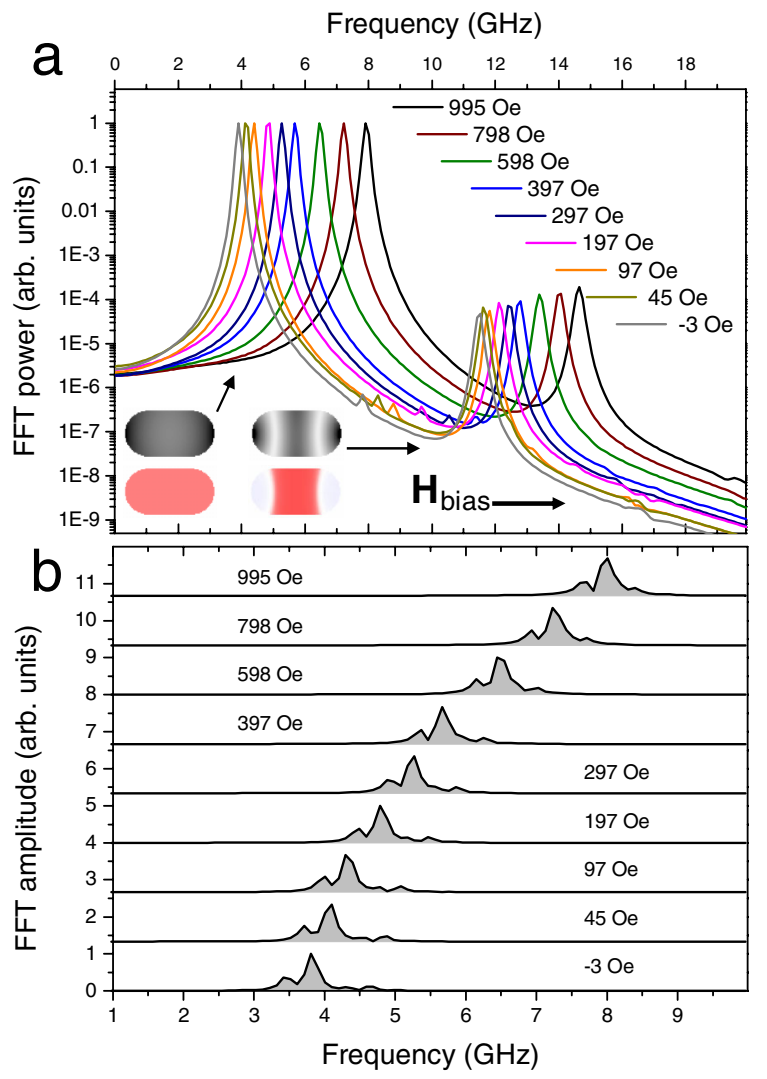

FIG. 2 (color online). Simulated FFT spectra of the out-ofplane component of the average dynamic magnetization of (a) an isolated nanoelement and (b) the center nanoelement within a $3 \times 3$ array are shown for different values of the bias magnetic field. The inset in (a) shows simulated magnitude (top) and phase (bottom) profiles for the modes observed at the bias field of 197 Oe. 


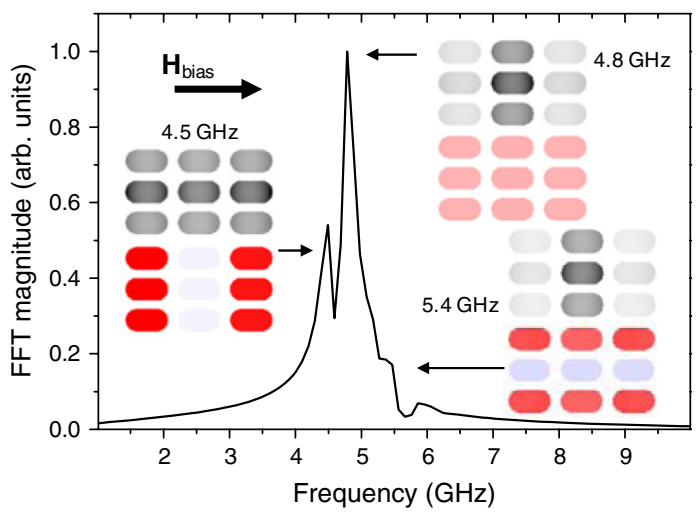

FIG. 3 (color online). Simulated images of the magnitude (top) and phase (bottom) for the modes at $4.5 \mathrm{GHz}, 4.8 \mathrm{GHz}$, and $5.4 \mathrm{GHz}$ of the $3 \times 3$ array are shown for a bias field of $197 \mathrm{Oe}$.

different peaks look the same in the center element. However, a remarkable difference is observed when the $3 \times 3$ array is considered as a whole. At the frequency of the highest peak $(4.8 \mathrm{GHz})$, all elements precess in phase, while the amplitude of precession is increased in the center column, being largest in the center element. At the frequency of $4.5 \mathrm{GHz}$, the center column precesses out of phase with the rest of the elements, while the amplitude of precession is increased in the center row. At the frequency of $5.4 \mathrm{GHz}$, the center row precesses out of phase with the rest of the elements, while the amplitude of precession is increased in the center column, being largest in the center element. This allows us to ascribe the splitting observed in the simulations to collective nonuniform precessional modes of the $3 \times 3$ array. The mode at $4.8 \mathrm{GHz}$ can be classified as quasiuniform, while the modes at $4.5 \mathrm{GHz}$ and $5.4 \mathrm{GHz}$ are the backward volumelike and DamonEshbach-like modes, respectively.

The qualitative similarity between the measured and simulated spectra leads us to expect that the splitting observed in the experiment is due to formation of collective spin wave modes. To be detectable, the modes should have a wavelength that is greater than the optical spot size, i.e., in the present case they should extend throughout the entire $4 \mu \mathrm{m}$ array, and so we attempted to image the collective modes at a bias magnetic field of 197 Oe. The FFT power spectrum calculated from the corresponding TR signal is shown in Fig. 4. The spectrum was fitted to a Lorentzian 3-peak function so that the mode frequencies could be precisely determined. The images of the dynamic magnetization in the entire array were measured at fixed time delays with 20 ps steps within the first 2 ns after the excitation. The images corresponding to the modes identified from the fit were generated using the procedure described and used for the micromagnetic simulations. We also used a harmonic magnetic field phase locked to the laser to excite the sample at the frequency of a particular mode, as described, e.g., in Ref. [15]. The images of the dynamic magnetization were then directly acquired, and were nearly identical to those shown in Fig. 4 [23].



FIG. 4 (color online). The FFT power spectrum calculated from the TR signal acquired from the center of the array at a bias magnetic field of 197 Oe is shown on a logarithmic scale together with the fit to a Lorentzian 3-peak function. The inset shows images of the modes confined within the entire array and corresponding to the peak frequencies identified from the fit. The darker shades of gray correspond to greater mode amplitude.

The measured mode images shown in Fig. 4 reveal nonuniform patterns of complex character. The images at 3.0, 3.6, and $4.2 \mathrm{GHz}$ should be compared with the simulated images at $4.5,4.8$, and $5.4 \mathrm{GHz}$, respectively. One should note that the splitting observed in Figs. 1 and 4 is smaller than the linewidth of the peaks. Hence, if two collective modes are close in frequency, the image of each mode may contain a contribution from the other. The latter contribution is likely to have a phase that is different to that of the primary mode, which can result in rather complex images being generated. In particular, a significant contribution from the dominant (quasiuniform) collective mode $(3.6 \mathrm{GHz}$ ) should be expected to be present as a background in images of the nonuniform modes at 3.0 and $4.2 \mathrm{GHz}$. It is also possible that each of the observed peaks represents a convolution of a family of closely spaced nonuniform collective modes, which have not been resolved experimentally and are absent from the simulations due to the simulated array size being much smaller than that in the experiment.

Several factors should be considered when interpreting the images. First, the internal magnetic field experienced by different elements within the array is expected to vary, as in the case of continuous magnetic elements $[13,15,20,24]$. However, the size of the demagnetized regions of this particular array as a whole is expected to be of the order of the length of a single magnetic element. Hence, this nonuniformity cannot have any noticeable effect on the modes observed here.

Second, the mode frequency of individual elements (neglecting the coupling) could vary due to the inevitable presence of defects and dispersion in the size and/or shape of the nanoelements. However, due to the interelement coupling, the precessional dynamics of the array would still be described by the spectrum of its normal modes rather than by frequencies of individual elements. We 
note that the number of modes is determined by the number of elements in the array, and generally speaking, does not depend upon the frequencies of individual elements or the strength of interaction [1].

Third, the finite damping of spin waves has to be considered. If the spin wave propagation length was smaller than the size of the array, the precession within different regions of the array would not be correlated and the regions could in principle support different sets of normal modes. However, this is unlikely to apply to the present case, since the detected modes span different and yet overlapping areas, each extending across the entire array (Fig. 4).

Finally, the shape and symmetry of the modes have to be addressed. The regions of higher amplitude appear to be tilted relative the horizontal and vertical axes. This could be due to a nonuniform magnetic ground state formed within the array as a whole. Indeed, the magnetodipole coupling among elements within the array favors nonuniform ordering of their magnetic moments. In the absence of the exchange interaction between different elements, the bias magnetic field presents the only, and then relatively small, opposition to nonuniform alignment. This asymmetry further adds to the aforementioned complexity of the mode profiles. Hence, one can say that, with respect to both the long wavelength spin waves observed here and the microwave field leading to their excitation, the array acts as a "magnonic metamaterial". This finding complements the recent observations of the magnonic band gap, which similar arrays exhibit for spin waves with a wavelength of the order of the array period $[6,7]$.

In summary, we have observed splitting of precessional modes in a micrometer sized array of closely spaced magnetic nanoelements. Micromagnetic simulations confirmed that the interelement interaction is strong enough to promote formation of detectable collective spin wave modes of $3 \times 3$ arrays of such elements, while the experiments revealed that the collective modes extend through the entire measured array. The spin waves are confined within the array as if it was a single element made of a continuous material, properties of which were determined by the structure and geometry of the array and its constituent nanoelements. Thus, such arrays appear as tailored magnonic metamaterials to spin and electromagnetic waves with a wavelength much greater the period of the array.

The research leading to these results received funding from EPSRC of UK and the European Commission's 7th Framework Programme (GA No. 233552).

*V.V.Kruglyak@exeter.ac.uk

[1] L. D. Landau and E. M. Lifshitz, Mechanics (ButterworthHeinemann, Oxford, 1976).

[2] C. Kittel, Introduction to Solid State Physics (John Wiley \& Sons, New York, 1996).

[3] A. I. Akhiezer, V. G. Bar'yakhtar, and S. V. Peletminskii, Spin Waves (North-Holland, Amsterdam, 1968).
[4] S. Jung et al., Phys. Rev. B 66, 132401 (2002); G. N. Kakazei et al., J. Appl. Phys. 93, 8418 (2003); G. Gubbiotti et al., J. Phys. Condens. Matter 16, 7709 (2004); M. Pardavi-Horvath, C. A. Ross, and R.D. McMichael, IEEE Trans. Magn. 41, 3601 (2005); F. Giesen et al., Phys. Rev. B 75, 184428 (2007); A. Barman et al., Nano Lett. 6, 2939 (2006).

[5] V. V. Kruglyak et al., Phys. Rev. B 71, 220409(R) (2005); V. V. Kruglyak et al., ibid. 75, 024407 (2007); P. S. Keatley et al., ibid. 78, 214412 (2008).

[6] G. Gubbiotti et al., J. Appl. Phys. 99, $08 C 701$ (2006); L. Giovannini, F. Montoncello, and F. Nizzoli, Phys. Rev. B 75, 024416 (2007).

[7] G. Gubbiotti et al., Appl. Phys. Lett. 90, 092503 (2007); Z. K. Wang et al., Appl. Phys. Lett. 94, 083112 (2009).

[8] O. Hellwig et al., Appl. Phys. Lett. 90, 162516 (2007).

[9] A. Slavin and V. Tiberkevich, IEEE Trans. Magn. 45, 1875 (2009), and references therein.

[10] R. E. Camley, T. S. Rahman, and D. L. Mills, Phys. Rev. B 27, 261 (1983); P. Grünberg and K. Mika, ibid. 27, 2955 (1983); S. A. Nikitov, P. Tailhades, and C.S. Tsai, J. Magn. Magn. Mater. 236, 320 (2001); M. Krawczyk and H. Puszkarski, Phys. Rev. B 77, 054437 (2008).

[11] J. O. Vasseur et al., Phys. Rev. B 54, 1043 (1996); V. V. Kruglyak and R. J. Hicken, J. Magn. Magn. Mater. 306, 191 (2006); S. Neusser and D. Grundler, Adv. Mater. 21, 2927 (2009).

[12] W. K. Hiebert, A. Stankiewicz, and M. R. Freeman, Phys. Rev. Lett. 79, 1134 (1997).

[13] A. Barman et al., Appl. Phys. Lett. 82, 3065 (2003).

[14] M. Buess et al., Phys. Rev. Lett. 93, 077207 (2004).

[15] S. Tamaru et al., Phys. Rev. B 70, 104416 (2004).

[16] Magnetic properties of the material were reported in Ref. [17], where it was found that the multilayer material in the frequency range of interest behaves as a single magnetic layer with a thickness of $4.6 \mathrm{~nm}$ and a magnetization of $760 \mathrm{emu} / \mathrm{cm}^{3}$. The uniaxial and surface anisotropy constants were found to be $16300 \mathrm{erg} / \mathrm{cm}^{3}$ and $0.254 \mathrm{erg} / \mathrm{cm}^{2}$, respectively, with the easy axis canted at $78^{\circ}$ from the direction of the CPW.

[17] P. S. Keatley et al., J. Appl. Phys. 105, $07 D 308$ (2009).

[18] M. Donahue and D.G. Porter, Object-oriented Micromagnetic Framework User's guide, Version 1.0, Interagency Report NISTIR 6376, NIST, 1999, http:// math.nist.gov/oommf.

[19] Simulations of much larger, if not complete, arrays would be required to precisely reproduce the dynamic behavior observed experimentally. This was impossible due to computational restrictions. Simulations of larger but yet small enough to be computationally affordable arrays would bring into consideration the array's edge effects that were not detectable experimentally here.

[20] J. P. Park et al., Phys. Rev. Lett. 89, 277201 (2002).

[21] R. D. McMichael and M.D. Stiles, J. Appl. Phys. 97, 10J901 (2005).

[22] N. Smith et al., IEEE Trans. Magn. 42, 114 (2006).

[23] See supplementary material at http://link.aps.org/ supplemental/10.1103/PhysRevLett.104.027201 for images of the dynamic magnetization acquired using cw excitation.

[24] H. Puszkarski, M. Krawczyk, and J.-C. S. Levy, Phys. Rev. B 71, 014421 (2005). 